\title{
Functional outcome of operatively treated displaced intra-articular calcaneal fractures using two parallel contoured reconstruction plates
}

\author{
Lakhey $\mathrm{S}^{1}$, Manandhar $\mathrm{RR}^{2}$, Pradhan $\mathrm{RL}^{3}$, Pandey $\mathrm{BK}^{2}$, Sharma $\mathrm{S}^{4}$, Rijal KP \\ ${ }^{1}$ Associate Professor, ${ }^{2}$ Lecturer, ${ }^{3}$ Assistant Professor, ${ }^{4}$ Senior Resident, ${ }^{5}$ Professor, Department of Orthopaedics, \\ Kathmandu Medical College, Sinamangal, Nepal
}

\begin{abstract}
Introduction: The treatment of displaced intra-articular calcaneal fracture is controversial. Conventionally, they were treated non-operatively. However, some surgeons are now operatively treating these fractures because of continuing dissatisfaction with the outcome of conservative treatment of these fractures and improvements that have occurred in surgical techniques and complication rates.

Objective: The aim of this study was to determine the functional outcome of operatively treated displaced intra-articular calcaneal fractures using two parallel contoured reconstruction plates.

Materials and methods: 12 patients with 14 displaced intra-articular calcaneal fractures involving the subtalar joint were included in the study conducted between July 2005 and December 2008. The fracture site was exposed using extended lateral approach. Internal fixation was done by two nearly parallel $3.5 \mathrm{~mm}$ reconstruction plates and screws contoured to form a gentle curve in all cases with the first plate fixed just below the articular surface. At the end of follow up, the patients' foot function was assessed by Calcaneal Fracture Scoring System of Kerr et al. Patients were also enquired about their satisfaction with their treatment outcome.

Results: The patients were followed up for duration of 12 to 24 months (mean 15.64 months). The outcome score as measured by Calcaneal Fracture Scoring System ranged from 48 to 94 (mean 83.64). 11 of 12 patients (91.6 \%) were satisfied with the treatment.

Conclusion: Displaced intra-articular fractures treated by open reduction and internal fixation, using two nearly parallel, contoured reconstruction plates through an extensile lateral approach and following the principles of treatment of intraarticular fractures, have good functional results with high patient satisfaction rate.
\end{abstract}

Key words: displaced intra-articular calcaneal fractures, operative treatment.

$\mathrm{T}$ he treatment of displaced intra-articular calcaneal fracture (DIACF) is controversial. Historically, displaced intra-articular calcaneal fractures were treated non-operatively as surgery was not well developed ${ }^{1,2,3}$. Surgery was associated with significant incidence of wound complications, particularly sepsis ${ }^{1}$. However, non-operative treatment of DIACF was associated with long term symptoms of pain and deformity ${ }^{1}$. Hence, some authors advocated surgery ${ }^{4,5,6,7}$ because both patients and surgeons were unhappy with results of conservative method of treatment. In the 1980s and $90 \mathrm{~s}$, the opinion swing has been towards operative intervention. This reflects continuing dissatisfaction with the outcome of conservative treatment of these fractures and improvements that have occurred in surgical techniques and complication rates.

\section{Objective}

The aim of this study was to determine the functional outcome of operatively treated displaced intra-articular calcaneal fractures using two parallel contoured reconstruction plates.

\section{Materials and methods}

This was a prospective study involving patients with displaced intra-articular calcaneal fractures (DIACFs) involving the subtalar joint presenting between July 2005 and December 2008 had x-rays of the injured calcaneum: lateral and axial views.

The decision to operate was based on any one or more of the following findings outlined by Barie et $\mathrm{al}^{8}$ :

1. incongruous sub-talar joint with more than $2 \mathrm{~mm}$ displacement.

2. depression of the talus into horizontal position

Correspondence

Dr. Shishir Lakhey

Associate Professor, Department of Orthopaedics

Kathmandu Medical College, Sinamangal, Nepal

E-mail: slakhey64@yahoo.com 
3. gross displacement of the heel with sub-fibular impingement

4. bony pressure points likely to ulcerate or give mechanical pain.

Exclusion criteria for the patients were as follows.

1. age more than 65 years

2. open calcaneal fractures

3. peripheral vascular disease.

Twelve patients with 14 DIACFs which fitted the criteria were included in the study.

A CT scan of the calcaneum, including a 3D reconstruction image, was done in all patients planned for surgery to better understand the fracture pattern.

\section{Surgical Technique}

The patients were taken up for surgery after the skin over the heel started wrinkling. They were kept in the lateral position on the operating table with the affected limb up. High thigh pneumatic tourniquet was used and the fracture site was exposed using extended lateral approach ${ }^{9}$. The displaced fragments were reduced and held temporarily with k-wires. Corticocancellous bone graft or modified hydroxyappatite pellets (G-bone) was used to fill the sub-articular void after fracture reduction, if the surgeon thought it was necessary. Internal fixation was done by $3.5 \mathrm{~mm}$ reconstruction plates and screws. Two plates contoured to form gentle curves and placed in near parallel fashion were used in all cases with the first plate fixed just below the articular surface.

Wound closure was done over $10 \mathrm{G}$ negative suction drain in layers. A below knee plaster slab was applied. The drain was removed in 48 hours and the skin stitches in 2 weeks. Ankle mobilisation was started after the plaster slab was discarded in 3 weeks. The patients were followed up at 6 weeks, 3 months, 6 months, 12 months and 24 months time in the OPD. Partial weight bearing was allowed in six weeks time and full weight bearing on the affected limb was allowed after 3 months post-op. At the end of follow up ranging from 12 to 24 months, the patients' foot function was assessed by Calcaneal Fracture Scoring System of Kerr et al ${ }^{\mathbf{1 0}}$. Patients were also enquired about their satisfaction with their treatment outcome.

Results

The age of the patients ranged from 16 to 62 years (mean: 34.33 years). 11 patients were males and 1 was a female. The right foot was affected in 5 patients, the left in 5 and bilateral feet in 2 patients. Seven of the patients were farmers, 2 were labourers, 1 was a student, 1 was a painter and 1 was housewife. All the patients had sustained this injury as result of fall from height.

The patients were operated 10 to 15 days (mean 11.86 days) after injury. Cortico-cancellous bone graft was used in 4 and G-bone in 6 of the operated calcanei. 4 of the calcanei did not require bone graft or G-bone. Two of the heels developed superficial wound infection which healed with dressings and antibiotics. One patient developed deep infection which required rotation flap cover and removal of implant after 3 months. The patients were followed up for duration of 12 to 24 months (mean 15.64 months). The treatment outcome as measured by Calcaneal Fracture Scoring System ranged from 48 to 94 (mean 83.64). 11 of 12 patients $(91.6 \%)$ were satisfied with the treatment. None of the patients required sub-talar fusion till the last follow-up.

Table 1: Calcaneal fracture scoring system

\begin{tabular}{|l|l|l|}
\hline Maximum Points 100 & At rest & On activity \\
\hline Pain (36 points) & 18 & 18 \\
\hline None & 12 & 12 \\
\hline Slight & 6 & 6 \\
\hline Moderate & 0 & 0 \\
\hline Severe & \multicolumn{2}{|l|}{} \\
\hline Work (25 points) & 25 \\
\hline No change in job & 16 \\
\hline Modification of job & 8 \\
\hline Enforced change of job & 0 \\
\hline Unable to work & 25 \\
\hline Walking (25 points) & 16 \\
\hline No change in walking ability & 25 \\
\hline Minimal restriction & 8 \\
\hline Moderate restriction & 0 \\
\hline Severe restriction & 14 \\
\hline Walking aids (14 poionts) & 10 \\
\hline None & 6 \\
\hline Occasional stick & 3 \\
\hline Constant stick & 0 \\
\hline 1 stick & \\
\hline Crutches & \\
\hline
\end{tabular}




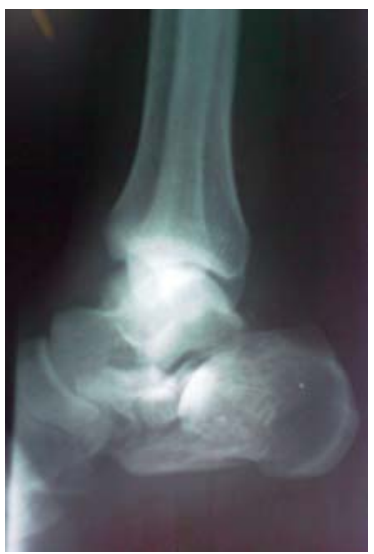

Fig 1: X-ray calcaneum lateral view

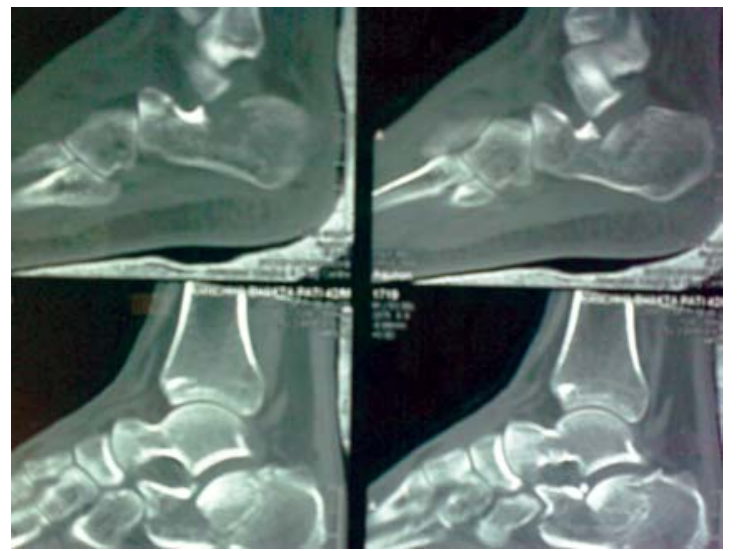

Fig 2: CT scan calcaneum sagittal cuts

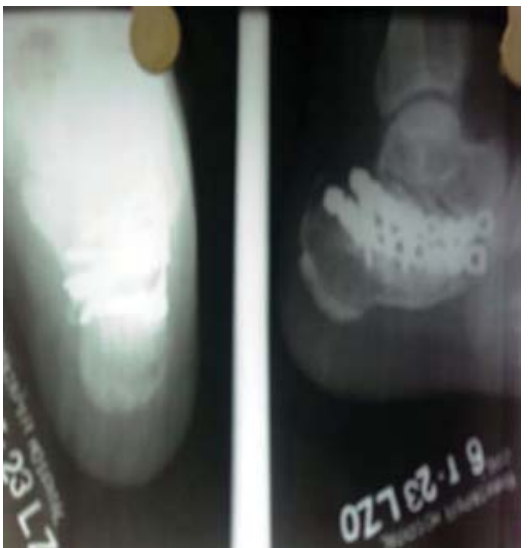

Fig 3: Post-op X-rays of calcaneum

\section{Discussion}

The management of every aspect of intra-articular calcaneal fractures is controversial. There are many systems for classifying DIACF, but there is no consensus amongst surgeons as to which is the most practical one. Opinion is divided as to whether operative treatment is better than conservative treatment. Opinion is also divided as to whether bone graft is required to fill the resultant sub-articular void after fracture reduction. Finally, amongst the many scoring system available to express treatment outcome, there is no consensus among surgeons as to which is the most scientific and practical system.

Essex-lopressti, Rowe and Sanders are the commonly used classification systems for calcaneal fractures. There is varying degrees of agreement among users of these classification systems. Although, classifications show positive correlation with outcome, there is no correlation with choice of treatment ${ }^{11,12,13}$. Therefore, the indication for surgery was not based on any classification system, but rather on the indications outlined by Barei et $\mathrm{al}^{8}$.

While some long term retrospective studies of nonoperatively treated intra-articular fractures have shown long term symptoms, ${ }^{1,14,15}$ other studies have reported relatively satisfactory long term results in severely displaced fractures managed conservatively $y^{3,15,16}$.

Historic cohort studies ${ }^{17,18,19}$ have suggested equal clinical outcomes with operative and conservative treatment of displaced intra-articular calcaneal fractures. While some of the more recent studies ${ }^{20,21,22}$ have also shown no advantage of operative treatment, many other studies ${ }^{23,24,25,26,27,28}$ have shown superior results with operative treatment. Earlier, surgical treatment was associated with significant incidence of wound complications, particularly sepsis ${ }^{1}$. However, conservative treatment is not without its complications of subtalar joint pain, heel varus and peroneal tendon impingement ${ }^{29}$.

Three to six percentage of patients with displaced calcaneal fractures treated operatively undergo subtalar joint fusion in future $23,30,31$ whereas non-operatively treated fractures are 5.5 to 6 times more likely to undergo subtalar joint fusion ${ }^{30,32}$. Better functional outcome with decreased wound complication, after sub-talar joint fusion, is seen in operatively treated displaced intraarticular calcaneal fractures ${ }^{33}$.

The principle of treating intra-articular fractures especially in a weight bearing joints are anatomical reduction, rigid fixation of intra-articular fractures and early mobilisation for good treatment outcome. ${ }^{34}$ Application of these principles to intra-articular calcaneal fractures have been slow because of complex bony and fracture anatomy, tenous soft tissue envelope and difficulty of achieving anatomic reduction and rigid fixation ${ }^{8}$ Improvements that have occurred in surgical techniques and complication rates have made many surgeons more operative in the treatment of these fractures.

These were the rationale for us deciding to treat DIACF operatively.

Open calcaneal fracture surgery can be performed using medial, lateral or combined approaches $7,9,35,36,37,38,39$. The lateral approach is the most popular approach. A lateral extensile exposure popularised by Benirschke and Sangeorzan ${ }^{9}$ was used in all our cases.

Various fixation devices like pelvic reconstruction plates $^{8}$, calcaneal plates ${ }^{34,40}, \mathrm{~K}$-wires ${ }^{40}$ or a combination 
of $\mathrm{k}$-wires and screws ${ }^{41}$ can be used for fixing these fractures. We used a combination of two $3.5 \mathrm{~mm}$ reconstruction plates and screws to fix these fractures. The two $3.5 \mathrm{~mm}$ reconstruction plates were contoured to form gentle curves and placed in near parallel fashion with the first plate fixed just below the articular surface. The "blow out" of the lateral wall, when present, could be well reduced and held in place with a combination of these two plates. The first contoured plate kept just below the articular surface fixed the fracture and supported the fractured articular surface whereas the second plate contoured and applied almost parallel to the first strengthened the fracture fixation of the first plate. The fixation afforded by the combination of these two nearly parallel plates is more surgeon friendly in terms of application and versatility as compared to the calcaneal plate. It also gives a better fracture fixation as compared to plain $\mathrm{K}$-wires or a combination of $\mathrm{K}$-wires and screws. To the best of our knowledge, this method of fixation has not been described in literature.

The indication for using bone graft is controversial ${ }^{42}$. As with other intra-articular fractures, bone graft should be used when the area beneath the posterior facet is vacant and non-supportive or whenever enough bone is missing or impacted that a buttress is needed ${ }^{7}$. However, various authors ${ }^{43,44}$, in recent studies have found no objective radiographic or functional benefits to the case of bone graft supplementation in the operative treatment of these fractures. We filled the resultant sub-articular void which occurred after fracture reduction, if it was substantial, with bone graft in the initial 4 cases and G-bone in the subsequent 6 cases. In 4 cases, it did not require any filling up. This is following the principles of bone grafting in articular fractures when there is a bony defect in the sub-articular region after fracture reduction. We changed from bone grafting the subarticular void in the earlier cases to filling it up with G-bone in the later cases to avoid donor site morbidity.

Outcome measurements can be expressed by various scoring systems $s^{18,45,46,47,48}$ or its modifications based on the author's experience of important symptoms and functional abilities. Kerr et $\mathrm{al}^{10}$ designed his scoring system by selecting the most relevant variables derived from other authors' judgement and experience of previous systems ${ }^{18,45,46,47,48}$. We chose the scoring system of Kerr et $\mathrm{al}^{10}$ for our study because it has been devised by scientific means and subjected to critical statistical analysis. Thus, the tendency of overestimation of outcome or inability to utilise the available range of scores and inability to distinguish between the patients at the most severe end of the range could be overcome increasing the overall sensitivity of the score using this scoring system. The ideal scoring system should span the full range of available scores and should have an approximately linear relationship with the ranked patient. The median score should be close to $50 \%$ of the available score. Kerr's calcaneal fracture scoring system comes closest to this amongst all the scoring systems available. We have chosen this system because it is concise, simple, scientific and easily reproducible.

Calcaneal Fracture Scoring System has been used by various other authors in recent publications for outcome measurements of treatment of calcaneal fractures. In a study conducted by Tennent et $\mathrm{al}^{23}$ a mean calcaneal fracture score of 87.7 for unilateral calcaneal fractures and 70.5 for those with bilateral calcaneal fractures or a contralateral foot/ankle injuries was achieved for operative treatment. Ibrahim et $\mathrm{al}^{20}$ achieved a mean score of 70.1 with conservative treatment and 63.5 with operative treatment. The difference in the scores was not statically significant $(\mathrm{p}=0.41)$. Potter et $\mathrm{al}^{31}$ reported operatively treated fractures with a mean score outcome of 70.0 in those who had sustained the injury as a result of fall and 61.3 in those with road traffic accident.

At the end of a mean of 15.64 months follow-up, we observed a mean Calcaneal Fracture Scoring System score of 83.64 in our patients. Our average score of 83.64 is comparable to that of Tennet et al and slightly higher than that of Ibrahim et al and Potter et al. We believe we were able to achieve a good mean Calcaneal fracture Score 83.64 because of our surgical technique and treatment protocol.

One patient developed deep wound infection which required rotation flap cover and implant removal. At the end of one year follow-up, her score was 48. She will probably require sub-talar joint fusion sometime in the future. 11 of 12 patients $(91.6 \%)$ were satisfied with the outcome of treatment.

There were certain limitations to our study. 12 patients with 14 calcanei were operated and their functional outcome score was measured at a mean follow-up of 15.64 months (range: 12-24 months). A study involving more patients followed up for a longer period of time can more accurately define the functional outcome of DIACF treated by this method.

\section{Conclusion}

Displaced intra-articular fractures treated by open reduction and internal fixation, using two nearly parallel, contoured reconstruction plates through an extensile lateral approach and following the principles of treatment of intra-articular fractures, have good functional results with high patient satisfaction rate. 
References

1. Lindsay R, Dewar F. Fracture of the os calcis. Am J Surg. 1958; 95: 555-76.

2. Rowe C, Sakellarides H, Freeman P, Sorbie C. Fractures of the os calcis. JAMA. 1963; 184: 920-3.

3. Pozo JL, Kirwan EO, Jackson AM. The longterm results of conservative management of severely displaced fractures of the calcaneus. JBJS(B). 1984; 66: 386-90.

4. Conn HR. The treatment of fractures of the os calcis. JBJS. 1935; 17: 392-405.

5. Essex-Lopresti P. The mechanism, reduction, technique and results in fractures of os calcis. Br J Surg. 1952; 39: 395-419.

6. Gallie WE. Subastragalar arthrodesis in fractures of the os calcis. JBJS. 1943; 24: 731-6.

7. Palmer I. The mechanism and treatment of fractures of the calcaneus. Open reduction with the use of cancellous grafts. JBJS(A). 1948; 30: 2-8.

8. Barei DP, Bellabarba C, Sangeorzan BJ, Benirschke SK. Fracture of the Calcaneus. OCNA. 2002; 33 (1): 263-85.

9. Benirschke SK, Sangeorzan BJ. Extensive intra-articular fractures of the foot: Surgical management of calcaneal fractures. Clin Orthop. 1993; 292: 128-34.

10. Kerr PS, Prothero DL, Atkins RM. Assessing outcome following calcaneal fracture: a rational scoring system. Injury. 1996; 27(1): 35-8.

11. Schepers T, van Lieshout EM, Ginai AZ, Mulder PG, Heetveld MJ, Patka P. Calcaneal fracture classification: a comparative study. J Foot Ankle Surg. 2009; 48(2): 156-62.

12. Humphrey CA, Dirschl DR, Ellis TI. Interobserver reliability of a CT-based fracture classification system. J Orthop Trauma. 2005; 19(9): 616-22.

13. Bhattacharya R, Vassan UT, Finn P, Port A. Sanders classification of fractures of the os calcis. An analysis of inter- and intra-observer variability. JBJS(B). 2005;87(2): 205-8.

14. Kitaoka HB, Schaap EJ, Chao EY etal: Displaced intra-articular fractures of the calcaneus treated non-operatively: Clinical results and analysis of motion and ground reaction and temporal forces. JBJS(A). 1994; 76: 1531-40.

15. Low CK, Mesenas S, Lam KS. Results of closed intra-articular calcaneal fractures treated with early mobilisation and without reduction. Ann Acad Med Singapore. 1995;24:820-22.
16. Salama R, Benamara A, Weissman L. Functional treatment of intra-articular fractures of the calcaneus. Clin Orthop. 1976;115:236-40.

17. Jarvholm U, Korner L, Thoren $\mathrm{O}$ and Wiklund LM. Fractures of the calcaneus. A comparision of open and closed treatment. Acta. Orthop. Scand. 1984; 55: 652-6.

18. Buckley RE, Meek RN. Comparison of open versus closed reduction of intraarticular calcaneal fractures: a matched cohort in workmen. J Orthop Trauma. 1992;6:216-22.

19. Kundel K, Funk E, Brutscher M, Bickel R. Calcaneal fractures: operative versus nonoperative treatment. J Trauma. 1996;41:83945.

20. Ibrahim $\mathrm{T}$, Rowsell M, Rennie W, Brown AR, Taylor GJS, Gregg PJ. Displaced intra-articular calcaneal fractures: 15-year follow-up of a randomised controlled trial of conservative versus operative treatment. Injury. 2007;38(7):848-55.

21. Parmar HV, Triffitt PD, Gregg PJ. Intra-articular fractures of the calcaneum treated operatively or conservatively. A prospective study. JBJS(B) 1993;75:932-7.

22. O'Farrell DA, O'Byrne JM, McCabe JP, Stephens MM. Fractures of the os calcis: improved results with internal fixation. Injury. 1993;24:263-5.

23. Tennent T, Calder PP, Salibury RD, Allen PW, Eastwood DM. The operative management of displaced intra-articular fractures of the calcaneum: a two-center study using a defined protocol. Injury. 2001;32:491-6.

24. Randle JA, Kreder HJ, Stephen D, Williams J, Laglal S, Hu R. Should calcaneal fractures be treated surgically? A Metaanalysis. Clin Orthop 2000;377-217.

25. Bridgman Sa, Dunn Km, Mcbride DJ, Richards PJ. Interventions for treating calcaneal fractures. Cochrane Database Sys Rev. 2000. CD001: 161.

26. Thordarson DB, Kreiger LE, Operative vs nonoperative treatment of intra-articular fractures of the calcaneus: a prospective randomised trial. Foot Ankle Int 1996; 17: 2-9.

27. Sanders R. Intra-articular fractures of the calcaneus: present state of the art. J Orthop Trauma. 1992; 6: 252-65.

28. Sanders R. Displaced intra-articular fractures of the calcaneus. JBJS(A). 2000; 82: 225-50. 
29. Myerson M, Quill GE. Late complications of fractures of the calcaneus. JBJS(A). 1993;75(3): z31-41.

30. Buckley R, Tough S, McCormack R, Pate G, Leighton R, Petrie D, Galpin R. Operative compared with non-operative treatment of dispalced intra-articular calcaneal fractures: a prospective, randomised, controlled multicentric trial. JBJS(A) 2002;84:1733-44.

31. Potter MQ, Nunley JA. Long-term functional outcomes after operative treatment for intraarticular fractures of the calcaneus. JBJS(A). 2009;91(8):1854-60.

32. Csizy M, Buckley R, Tough S, Leighton R, Smith J, McCormack R, Pate G, Petrie D, Galpin R. Displaced intra-articular calcaneal fractures: variables predicting late subtalar fusion. J Orthop Trauma. 2003;17(2):106-12.

33. Radnay CS, Clare MP, Sanders RW. Subtalar fusion after displaced intra-articular calcaneal fractures: does initial operative treatment matter? JBJS(A). 2009 ;91(3):541-6

34. Muller ME, Allgower M, Schneider R. Manual of Internal Fixation. Techniques recommended by AO Group. 2nd edition. New York: Springer;979. P. 71-87.

35. Burdeaux BD. Reduction of calcaneal fractures by the McReynolds medial approach technique and its experimental basis. Clin Orthop. 1983;177: 87-103.

36. Letournel E. Open reduction and internal fixation of calcaneus fractures. In Spiegel PG (editors). Topics in Orthopaedic Trauma. Baltimore: University Park Press; 1984. P. 17392.

37. McReynolds IS. The case for operative treatment of fractures of calcaneus. In Leach RE, Hoaglund FT, Riseborough EJ (eds): Controversies in Orthopaedic Surgery. Philedelphia: WB Saunders; 1982. P. 232-54.

38. Stephenson JR. Surgical treatment of displaced intra-articular fractures of the calcaneus: a combined lateral and medial approach. OCNA. 1993; 290: 68-75.
40. Stephenson JR. Surgical treatment of displaced intra-articular fractures of the calcaneus using medial and lateral approaches, internal fixation and early motion. JBJS(A). 1987;69:115-30.

40. Li X, Li Q, Zhang Z, Wen X, Yan H. Treatment of intra-articular calcaneal fractures using Kirschner's wire or calcaneal plate. Zhongguo Xiu Fu Chong Jian Wai Ke Za Zhi. 2008;22(4):459-62.

41. Di Schino M, Bensaïda M, Vandenbussche E, Augereau B, Nich C. Results of open reduction and cortico-cancellous autograft of intra-articular calcaneal fractures according to Palmer. Rev Chir Orthop Reparatrice Appar Mot. 2008;94(2):135-44.

42. Sanders R, Gregory P. Operative treatment of intra-articular fractures of the calcaneus. OCNA. 1995;26:203-14.

43. Tufescu TV, Buckley R. Age, gender, work capability and worker's compensation in patients with displaced intraarticular calcaneus fractures. J Orthop Trauma. 2001. 15:275-9.

44. Sanders R, Fortin P, DiPasquale $\mathrm{T}$, et al: Operative treatment in 120 displaced intraarticular fractures: Results using a prognostic computed tomography scan classification. Clin Orthop. 1993;290:87-95.

45. Rowe CR, Sakellarides HT, Freeman PA and Sorbic C. Fracture of the os calcis: a long term follow up study of 416 patients. JAMA. 1963;184: 920.

46. Crosby LA and Fitzgibbons T. Computerised tomographic scanning of acute intra-articuiar calcaneal fractures. JBJS(A). 1990;72A:852-9.

47. Paley D and Hall H. Intra-articular fractures of the calcaneus: a critical analysis of results and prognostic factors. JBJS(A). 1993; 75A:34254.

48. Sanders R, Fortin P, Dipasquale T and Walling A. Operative treatment of 120 displaced intraarticular fractures of the calcaneus. Clin Orthop. 1993;290: 87-95. 